\title{
A Community Grows around the Geysering World of Enceladus
}

\author{
Carolyn C. Porco
}

\begin{abstract}
The discovery by NASA's Cassini mission at Saturn in 2005 of a large plume of material erupting from the south polar terrain of Enceladus, sourced within a subsurface ocean of salty liquid water laced with organic compounds, has brought together scientists from a diverse range of disciplines over the last decade to evaluate this small moon's potential for extraterrestrial life. The collection of papers published today in Astrobiology, as the mission draws to a close, is the outcome of our most recent meeting at UC Berkeley in June 2016. Key Words: Enceladus-Enceladus Focus Group_Ocean world-Search for biosignatures. Astrobiology 17, 815-819.
\end{abstract}

$\mathbf{T}$ HE CASSINI MISSION's discovery, in the opening months of the year 2005, of a plume of icy particles (Porco et al., 2006) and vapor (Dougherty et al., 2006) erupting from the south polar region of the small saturnian moon Enceladus, along with follow-up observations collected later that year (e.g., Hansen et al., 2006; Spencer et al., 2006), led quickly to the suggestion that the phenomenon had its source in reservoirs of liquid water (Porco et al., 2006) laced with organic compounds (Waite et al., 2006). How deep the source region might lie below the surface was not then clear, but it was immediately obvious that, should we be right, we had the tangible expression of a body of water beyond Earth-perhaps one that could support life-within easy reach. The world took notice: the suggestion of an extraterrestrial habitable zone within Enceladus, inferred from Cassini's initial findings and published in Science in early 2006, reached the front page of the New York Times on March 10, 2006. By that time, it had become patently clear to me that a broad range of scientific disciplines would be required to address the question of whether or not biological activity existed within Enceladus.

So was born the idea to form an informal group of scientists from diverse fields of study who would meet from time to time to discuss the latest results and theories on Enceladus and, as a matter of course, set new research directions aimed at advancing our understanding of the moon's habitability and biological potential. Getting astrobiologists interested was the first step. I contacted Chris McKay at NASA Ames Research Center, who knew the astrobiology community better than I did, and requested his help in putting it together. He agreed. We sent out the word: Our first meeting would occur in the fall of 2006, on the campus of Caltech in Pasadena, California.

The vast majority of the attendees at that first meeting were planetary scientists of various stripes-geologists, geophysicists, spectroscopists, and dynamicists-as well as mission designers, all either offering results or ideas, or both, about how Enceladus might have become so geologically active today, or describing future missions and payloads that could expand on Cassini's discoveries. But I was especially pleased that we managed to collect a handful of attendees who began the work of wondering what kind of life, if any, might be present within Enceladus and how we might go about detecting it.

During the subsequent decade at Saturn, Cassini's remote sensing instruments extensively observed the plume and mapped the moon's surface. The plume was found to vary in mass on a diurnal timescale (Hedman et al., 2013); the phase of the variation was significantly delayed from what would be expected from widening and narrowing vents in response to varying tensional stresses in an elastic ice shell, but no model was conclusive (Nimmo et al., 2014). The spacecraft was flown through the plume about a

University of California, Berkeley, California.

Space Science Institute, Boulder, Colorado.

(c) Carolyn C. Porco, 2017; Published by Mary Ann Liebert, Inc. This Open Access article is distributed under the terms of the Creative Commons License (http://creativecommons.org/licenses/by/4.0), which permits unrestricted use, distribution, and reproduction in any medium, provided the original work is properly credited. 


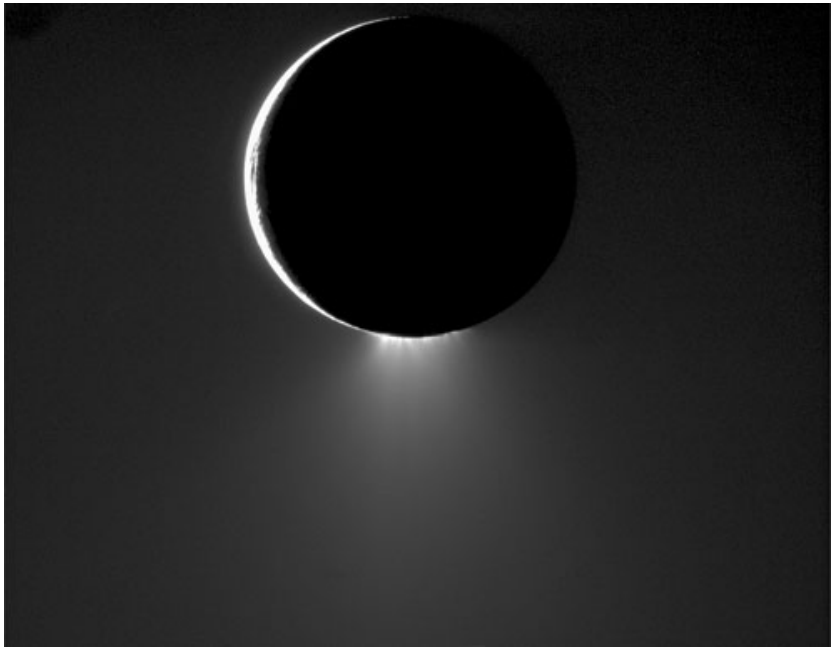

FIG. 1. An image of Enceladus and its southern geysers and the faint plume they form, taken by the Cassini high resolution camera on November 1, 2009 through a spectral filter centered at a wavelength of 752 nanometers from a distance of $\sim 209,000$ kilometers. The phase angle is $160^{\circ}$. The image scale is $\sim 1 \mathrm{~km} /$ pixel.

dozen times, allowing the in situ instruments to capture material and determine the composition of both ice particles and vapor (Postberg et al., 2009; Waite et al., 2009). In time, we learned that the source of Enceladus' $100+$ geysers that create the plume (Porco et al., 2014), as well as $\sim 5 \mathrm{GW}$ of thermal radiation (Spencer et al., 2013), all of which emerge from prominent fractures crossing the south polar terrain (SPT), is a salty, variable-thickness, global ocean some kilometers below the surface (Thomas et al., 2015) that reaches its greatest thickness under the SPT. Through these findings, together with more recent Cassini evidence of complex organic molecules (Postberg et al., 2017) and even seafloor hydrothermal activity driven by serpentinization (Hsu et al., 2015; Waite et al., 2017), the story of Enceladus has grown all the richer and has affirmed the moon's status as a prime candidate for astrobiological study and the search for life. No other ocean world in the Solar System beyond Earth has been studied in such depth and detail for so long, nor is any so well understood as Enceladus.

Our group continued to meet over the years at roughly 2year intervals, its membership and discipline representation changing with time. The number of people advising me grew also. By the time of our most recent gathering, in June 2016 on the campus of UC Berkeley, where I had become a visiting scholar in the Astronomy Department, our community had grown substantially. This was, by far, our most wide-ranging, cross-disciplinary meeting to date, and it was thrilling. The usual planetary and astrobiological sciences were well represented, but this time I had managed to lure to the subject organic chemists, microbiologists, microscopists, oceanographers, experts in microfluidics, those studying problems in the origin of life, and even one genomist from the UC Berkeley research lab responsible for the 2016 announcement of a new, far richer tree of life (Hug et al., 2016). Finally, our small, informal focus group had grown to become what I had originally hoped it would be: a place where the usual walls that grow and harden around different scientific disciplines had come crumbling down in the face of an irresistible and real opportunity to test life's distribution in the Cosmos.

The papers gathered together in this special collection devoted to Enceladus, with one exception, resulted from our Berkeley meeting. These works offer a glimpse into the state of a rapidly changing subject at one moment in time ... as the Cassini mission ends and we are contemplating the next steps in the exploration of this fantastic little moon. The opening two papers (Barge and White, 2017; Deamer and Damer, 2017) present the examination of two distinct issues, both of extreme importance to the origin of biochemisty. Barge and White focus on the chemistry in hydrothermal vent systems, like those we now believe may be present on the seafloor of Enceladus. These authors ask whether the ability of hydrothermal chimneys to maintain compositional and energy gradients might have played an important role in driving the origin of life and its metabolic characteristics. Deamer and Damer are not pursuing the origin of metabolism, but rather the origin of life's infrastructure and how the basic biomolecules, such as amino acids and other building blocks, could have been concentrated sufficiently to initiate the formation of more complex molecules. The Barge and White study says life requires biologically usable gradients and therefore was more likely to originate where there were plenty of them ... oceanic vents. The Deamer and Damer study says that life began when and where the proper building materials could be first assembled, such as hydrothermal springs on the surface that underwent natural cycles of hydration and dehydration. You can read and decide, if you wish, for yourself, or merely be amazed at how far we still are from having a theory for how life began.

The third paper (Benner, 2017) offers a somewhat unusual universal biosignature in the form of testable molecular characteristics that, the author argues, any information-bearing biopolymer must have. An initial proposal along these lines was first made by Schrödinger 70 years ago (Schrödinger, 1943): the various building blocks of a genetic polymer underlying a successful biochemistry must all be similar in size so that they form a regular "crystalline" structure that remains unchanged as the system evolves and the building blocks themselves are rearranged. The second feature, proposed by Benner over a decade ago (Benner and Hutter, 2002), expands on Schrödinger to note that in order to support Darwinian evolution, the physical/chemical behavior of a genetic biopolymer must also remain unchanged as the system evolves. This is achievable in water only if the biopolymer has a backbone with a repeating charge; that is, the biopolymer must be a "polyelectrolyte." The genetic biopolymers of terran life, that is, the nucleic acids composed of nucleotide building blocks, have such a feature: a backbone of repeating components - the phosphate group-each with an identical charge whose mutual repulsion stretches the molecules; discourages folding; allows templating; and makes it possible for their genetic information, as well as random mutations, to be reliably transcribed from generation to generation. It also allows the overall physical/chemical behavior to persist against structural changes, such as the rearrangement of the building blocks. Benner proposes 


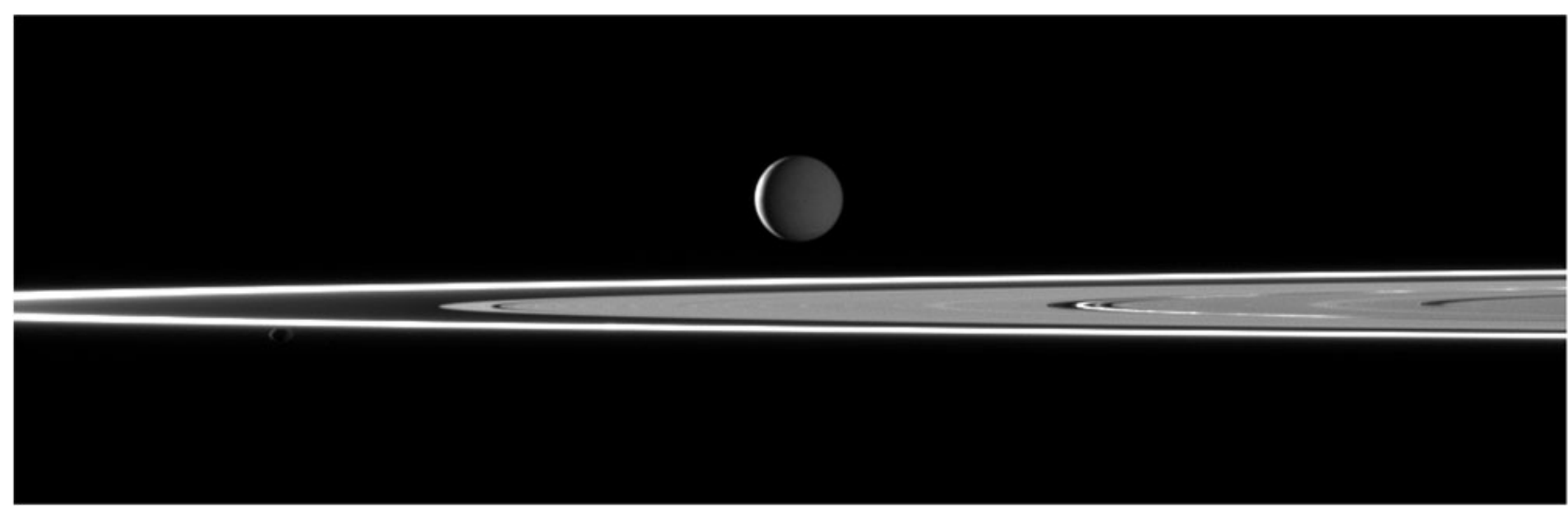

FIG. 2. Two light sources illuminate Enceladus in this image taken in visible light with the Cassini high resolution camera on February 11, 2010 from a distance of $\sim 1.5$ million kilometers and a phase angle of $142^{\circ}$. Most of the moon is dimly lit by sunlight reflected off Saturn. However, a thin crescent of the moon is directly lit by sunlight. Saturn's bright rings are in the foreground. Image scale is $\sim 9 \mathrm{~km} /$ pixel.

this as a requirement for any genetic polymer, regardless of the chemical constituents. The advantage of polyelectrolytes is that they are easily concentrated from dilute aqueous solution, like the Enceladus plume. This permits, in practice, the recovery of any universal Darwinian biopolymer using a device no more complicated than a polycharged surface. Maybe some mission back to Enceladus will someday carry such a capability.

The fourth paper (Judge, 2017) is the one contribution that did not arise from our Berkeley meeting. It dials back the ambition and expense of future explorations of Enceladus and suggests that, to some degree, the chemical makeup of the plume can be investigated, and the search for biomolecules can be conducted, by infrared spectroscopic and spectropolarimetric methods, that is, observing a plume erupting from a body as it transits a bright planetary disk like Saturn, from the largest ground-based telescopes and even eventually the James Webb telescope. Jupiter's $3^{\circ}$ obliquity ensures that Europa transits of Jupiter occur every europan day. Enceladus transits of Saturn, whose obliquity is a healthy $27^{\circ}$, are seasonal and next begin in 2022 . It is not too early to start making plans.

The four following papers in this collection address, in one form or another, the anticipated bioloads at Enceladus, and the detectable biosignatures that might be present in its plume. Steel et al. (2017) construct a thermal model that assumes $10 \%$ of the geothermal heat emerging from the moon's core drives hot $\left(90^{\circ} \mathrm{C}\right)$ hydrothermal fluid flow, which results, through water/rock interactions, in the production of $\mathrm{H}_{2}$. In their work, $100 \%$ of the molecular hydrogen thus produced is subsequently consumed by methanogens to produce biomass. They thus estimate, at the vents, $90 \mu M$ of biologically produced amino acids, and microbial concentrations as high as $10^{9}$ cells/mL; $10 \%$ of the latter rise in the thermal plumes that originate at the vents and eventually reach the base of the ice shell. If these authors are correct, and Enceladus approaches this highefficiency scenario, especially if the process of bubblescrubbing (see below) is at work, then the search for biosignatures, even microbes, in the samples collected from Enceladus' plume could be easily accomplished.
In the Porco et al. (2017) study, my coauthors and I photometrically reduce Cassini images to particle number densities at various altitudes in the plume and use the results to estimate the volume of a sample that future Enceladus missions would collect, ranging from single fly-through missions to landers. Like Steel et al. (2017), we estimate the bioload at the Enceladus seafloor vents, but unlike Steel et al., we merely scale the bioload at Lost City by the ratio of the geothermal energy flux through the moon's seafloor to that of Earth ... a ratio that turns out to be 1 . Our results, $\sim 10^{5}$ cells $/ \mathrm{mL}$, are significantly less optimistic than those of Steel et al. However, we introduce to the study of Enceladus, and describe in some detail, the process of bubblescrubbing, well known to marine microbiologists, whereby microbes and organic matter in natural bodies of water may be found in significantly enhanced concentrations in the spray produced at the surface by breaking bubbles. This outcome follows from the tendency of bubbles, rising in a column of water, to collect available organic material along the way. It is a process that could very well be at work on Enceladus and could make the job of detecting biosignatures within the plume up to a 1000 -fold easier.

Mathies et al. (2017) describe what can be done with very small sample sizes and microfluidics in determining the concentrations of organic materials in the plume and in determining whether or not particular molecular species show enantiomeric excess, an arguably strong indicator of life. Mathies, the lead author, is one of the earliest developers of microfabricated "lab-on-a-chip" technology and its application to space instruments. He and his coauthors make the case for the scientific feasibility of the Enceladus Organic Analyzer (EOA), a lab on a chip that uses a microfluidic capillary electrophoresis system to sensitively detect a wide range of relevant organic molecules, including amines, amino acids, carboxylic acids, aldehydes, ketones, and thiols with part-permillion sensitivity. The engineering design for both the chip and the capture plate to collect plume particles-not an easy task-are both presented. Now all we need is a spacecraft. And a big rocket. And a bit of funding.

Bedrossian et al. (2017) expand on their previous work (Wallace et al., 2015; Lindensmith et al., 2016) in 
designing and field testing an extraordinary microscopic system being developed at JPL and Caltech: the digital holographic microscope (DHM). True to its name, the DHM is a microscope capable of taking holographic images of a (comparatively) wide field of view, which is necessary for exploratory "survey" work, that capture phase as well as spatial information. The quantitative phase information can be used to distinguish unresolved organisms from bits of inorganic matter; one is made largely of water, the other is not, and so their different indices of refraction make them readily distinguishable. Also, the inclusion of video framing means that any motility would be a discriminating feature as well, made more powerful by the phase capability that allows detection of motion in the third, "out of plane" dimension. Obviously, for a microscope to be fruitful at imaging intact organisms, either plume fly-through speeds must be below $\sim 2 \mathrm{~km} / \mathrm{s}$ or the DHM would need to be placed on a lander. A video clip of alien organisms in motion could well be the only unambiguous, $100 \%$ confidence-level detection we gather of enceladan life, should it be there at all.

The final two papers in our collection make significant advances in the continued analysis of Cassini data and expand our basic knowledge of the geophysical mechanisms underlying the geysers. Teolis et al. (2017) attempt what, to date, has not been done: determine the threedimensional structure of the vapor component of the plume by comparing, in detail, the vapor measurements by the Cassini Ion and Neutral Mass Spectrometer and the Ultraviolet Imaging Spectrometer with the full, three-dimensional map of the 100+ geysers seen in ISS images of the particle component, with the intent to look for signatures of the latter in the former. Though obvious stochasticity in the vapor emissions makes the job tough, these authors nonetheless are able to say that the structure of the vapor component seems to be best fit with the many-geyser model with some contributions from continuous, inter-geyser emissions along the fractures (Porco et al., 2014). That both types of emissionsdiscrete and continuous eruptions - are seen in high-resolution Cassini images of the near-surface altitudes of the geysers is corroboration of this conclusion.

Finally, in all previous attempts to explain the diurnal variation of the plume by modeling the cyclical behavior of tidal stresses across the SPT (e.g., Hurford et al., 2013; Nimmo et al., 2014; Běhounková et al., 2015), none were implemented to account for the effect of the fractures themselves on the tidal stress field. The ice shell was treated as a solid, unbroken mass, and the stresses were merely computed at surface locations along the main, geyser-producing fractures and assumed to be the same below the surface. Běhounková et al. (2017) take a big step forward in their updated work presented here in devising a numerical method for decoupling one side of a fracture from the other in computing the time-variable stresses. The results are remarkable. Stresses and strains become significantly larger in the vicinity of the fractures, and their model-which still retains some rather simplifying assumptions, such as constant fault-geometry with depth, neglect of stress, friction, and liquid water within the fracture-predicts a spatial distribution of stresses significantly different than that of the nonfracture model. At the moment, the pattern of activity these authors predict does not match the Cassini observations (Porco et al., 2014) in satisfactory detail. But the work is still in its infancy, and I'm sure that, with such powerful new capabilities, it won't be long before this research group will elucidate those factors that are most important in determining the observed distribution of activity across the surface and the precise shape and phase of the plume's diurnal variation in mass.

So, now, I leave you to it. As Cassini's extraordinary 13 years of exploration conclude, enjoy this up-to-theminute, far-reaching, wide-ranging look at that little moon at Saturn with the big possibilities.

\section{References}

Barge, L.M. and White, L.M. (2017) Experimentally testing hydrothermal vent origin of life on Enceladus and other icy/ ocean worlds. Astrobiology 17:820-833.

Bedrossian, M., Lindensmith, C., and Nadeau, J.L. (2017) Digital holographic microscopy, a method for detection of microorganisms in plume samples from Enceladus and other icy worlds. Astrobiology 17:913-925.

Běhounková, M., Tobie, G., Čadek, O., Choblet, G., Porco, C., and Nimmo, F. (2015) Timing of water plume eruptions on Enceladus explained by interior viscosity structure. Nat Geosci 8:601-604.

Běhounková, M., Souček, O., Hron, J., and Čadek, O. (2017) Plume activity and tidal deformation on Enceladus influenced by faults and variable ice shell thickness. Astrobiology 17:941-954.

Benner, S.A. (2017) Detecting Darwinism from molecules in the Enceladus plumes, Jupiter's moons, and other planetary water lagoons. Astrobiology 17:840-851.

Benner, S.A. and Hutter, D. (2002) Phosphates, DNA, and the search for nonterrean life: a second generation model for genetic molecules. Bioorg Chem 30:62-80.

Deamer, D. and Damer, B. (2017) Can life begin on Enceladus? A perspective from hydrothermal chemistry. Astrobiology 17:834-839.

Dougherty, M.K., Khurana, K.K., Neubauer, F.M., Russell, C.T., Saur, J., Leisner, J.S., and Burton, M.E. (2006) Identification of a dynamic atmosphere at Enceladus with the Cassini magnetometer. Science 311:1406-1409.

Hansen, C.J., Esposito, L., Stewart, A.I.F., Colwell, J., Hendrix, A., Pryor, W., Shemansky, D., and West, R. (2006) Enceladus' water vapor plume. Science 311:1422-1425.

Hedman, M.M., Gosmeyer, C.M., Nicholson, P.D., Sotin, C., Brown, R.H., Clark, R.N., Baines, K.H., Buratti, B.J., and Showalter, M.R. (2013) An observed correlation between plume activity and tidal stresses on Enceladus. Nature 500: 182-184.

Hsu, H.W., Postberg, F., Sekine, Y., Shibuya, T., Kempf, S., Horanyi, M., Juhasz, A., Altobelli, N., Suzuki, K., Masaki, Y., Kuwatani, T., Tachibana, S., Sirono, S.I., Moragas-Klostermeyer, G., and Srama, R. (2015) Ongoing hydrothermal activities within Enceladus. Nature 519:207-210.

Hug, L.A., Baker, B.J., Anantharaman, K., Brown, C.T., Probst, A.J., Castelle, C.J., Butterfield, C.N., Hernsdorf, A.W., Amano, Y., Ise, K., Suzuki, Y., Dudek, N., Relman, D.A., Finstad, K.M., Amundson, R., Thomas, B.C., and Banfield, J.F. (2016) A new view of the tree of life. Nat Microbiol 1, doi:10.1038/nmicrobiol.2016.48.

Hurford, T., Brunt, K.M., and Rhoden, A. (2013) Antarctic analog for diurnal tidal motions along fractures on Enceladus 
[abstract P53B-1866]. In AGU Fall Meeting, American Geophysical Union, Washington, DC.

Judge, P. (2017) A novel strategy to seek biosignatures at Enceladus and Europa. Astrobiology 17:852-861.

Lindensmith, C.A., Rider, S., Bedrossian, M., Wallace, J.K., Serabyn, E., Showalter, G.M., Deming, J.W., and Nadeau, J.L. (2016) A submersible, off-axis holographic microscope for detection of microbial motility and morphology in aqueous and icy environments. Plos One 11, doi:10.1371/journal .pone. 0147700 .

Mathies, R.A., Razu, M.E., Kim, J., Stockton, A., Turin, P., and Butterworth, A. (2017) Feasibility of detecting bioorganic compounds in Enceladus plumes with the Enceladus Organic Analyzer (EOA). Astrobiology 17:902-912.

Nimmo, F., Porco, C., and Mitchell, C. (2014) Tidally modulated eruptions on Enceladus: Cassini ISS observations and models. Astron J 148, doi:10.1088/0004-6256/148/3/46.

Porco, C., Helfenstein, P., Thomas, P.C., Ingersoll, A.P., Wisdom, J., West, R., Neukum, G., Denk, T., Wagner, R., Roatsch, T., Kieffer, S., Turtle, E., McEwen, A., Johnson, T.V., Rathbun, J., Veverka, J., Wilson, D., Perry, J., Spitale, J., Brahic, A., Burns, J.A., Del Genio, A.D., Dones, L., Murray, C.D., and Squyres, S. (2006) Cassini observes the active south pole of Enceladus. Science 311:1393-1401.

Porco, C., DiNino, D., and Nimmo, F. (2014) How the geysers, tidal stresses, and thermal emission across the south polar terrain of Enceladus are related. Astron J 148, doi:10.1088/ 0004-6256/148/3/45.

Porco, C.C., Dones, L., and Mitchell, C. (2017) Could it be snowing microbes on Enceladus? Assessing conditions in its plume and implications for future missions. Astrobiology 17:876-901.

Postberg, F., Kempf, S., Schmidt, J., Brilliantov, N., Beinsen, A., Abel, B., Buck, U., and Srama, R. (2009) Sodium salts in E ring ice grains from an ocean below the surface of Enceladus. Nature 459:1098-1101.

Postberg, F., Khawaja, N., Kempf, S., Waite, J.H., Glein, C., Hsu, H.W., and Srama, R. (2017) Complex organic macromolecular compounds in ice grains from Enceladus [abstract 1401]. In $48^{\text {th }}$ Lunar and Planetary Science Conference, Lunar and Planetary Institute, Houston.

Schrödinger, E. (1943) Was ist Leben, Serie Piper, Vol. 1134.

Spencer, J.R., Pearl, J.C., Segura, M., Flasar, F.M., Mamoutkine, A., Romani, P., Buratti, B.J., Hendrix, A.R., Spilker, L.J., and Lopes, R.M.C. (2006) Cassini encounters Enceladus: background and the discovery of a south polar hot spot. Science 311:1401-1405.
Spencer, J.R., Howett, C.J.A., Verbiscer, A., Hurford, T.A., Segura, M., and Spencer, D.C. (2013) Enceladus heat flow from high spatial resolution thermal emission observations [EPSC2013-840-1]. In EPSC Abstracts, European Planetary Science Congress.

Steel, E.L., Davila, A., and McKay, C.P. (2017) Abiotic and biotic formation of amino acids in the Enceladus ocean. Astrobiology 17:862-875.

Teolis, B.D., Perry, M.E., Hansen, C.J., Waite, J.H., Porco, C.C., Spencer, J.R., and Howett, C.J.A. (2017) Enceladus plume structure and temporal variability: comparison of Cassini observations. Astrobiology 17:926-940.

Thomas, P.C., Tajeddine, R., Tiscareno, M.S., Burns, J.A., Joseph, J., Loredo, T.J., Helfenstein, P., and Porco, C. (2015) Enceladus's measured physical libration requires a global subsurface ocean. Icarus 264:37-47.

Waite, J.H., Combi, M.R., Ip, W.-H., Cravens, T.E., McNutt, R.L., Kasprzak, W., Yelle, R., Luhmann, J., Niemann, H., Gell, D., Magee, B., Fletcher, G., Lunine, J., and Tseng, W.-L. (2006) Cassini Ion and Neutral Mass Spectrometer: Enceladus plume composition and structure. Science 311: 1419-1422.

Waite, J.H., Lewis, W.S., Magee, B.A., Lunine, J.I., McKinnon, W.B., Glein, C.R., Mousis, O., Young, D.T., Brockwell, T., Westlake, J., Nguyen, M.J., Teolis, B.D., Niemann, H.B., McNutt, R.L., Perry, M., and Ip, W.H. (2009) Liquid water on Enceladus from observations of ammonia and ${ }^{40} \mathrm{Ar}$ in the plume. Nature 460:487-490.

Waite, J.H., Glein, C.R., Perryman, R.S., Teolis, B.D., Magee, B.A., Miller, G., Grimes, J., Perry, M.E., Miller, K.E., Bouquet, A., Lunine, J.I., Brockwell, T., and Bolton, S.J. (2017) Cassini finds molecular hydrogen in the Enceladus plume: evidence for hydrothermal processes. Science 356:155-159.

Wallace, J.K., Rider, S., Serabyn, E., Kuhn, J., Liewer, K., Deming, J., Showalter, G., Lindensmith, C., and Nadeau, J. (2015) Robust, compact implementation of an offaxis digital holographic microscope. Optics Express 23: 17367-17378.
Address correspondence to: Carolyn Porco

E-mail: carolyn@ciclops.org

Submitted 19 June 2017 Accepted 30 June 2017 